\title{
RESEÑA: ESCRITORAS DE LA MODERNIDAD (1880-1920). LA \\ TRANSFORMACIÓN DEL CANON. MARÍA VICENTA HERNÁNDEZ ÁLVAREZ (COORD.) ${ }^{1}$
}

\author{
Angelo Azzilonna ${ }^{2}$
}

\section{María Vicenta Hernández Álvarez (Coord.). Escritoras de la modernidad (1880- 1920). La transformación del canon. Granada: Comares, 2018. ISBN 978-84-9045-} 752-8.

Ancora oggi, in pieno XXI secolo, benché sotto forme silenti e meno plateali rispetto al passato, persino nella civile e progredita Europa, le cronache giornalistiche ci informano di nefaste vicende e discriminazioni di genere che confermano il persistere di un inveterato modello socioculturale di tipo patriarcale, a sua volta, rivelatore della millenaria condizione di subalternità femminile e delle asimmetriche relazioni tra $\mathrm{i}$ sessi, dimostrando un'uguaglianza tra i sessi, teoricamente indiscutibile ma non sempre corroborata dalla pratica e dai fatti. A tal proposito si pensi ad esempio alle innumerevoli e quotidiane denunce di maltrattamenti, di violenza fisica e psicologica, patiti dalle donne o alla maggiore difficoltà e reticenza nei loro confronti nell'occupare posti rilevanti in ambiti lavorativi considerati più adatti all'uomo, quali la politica, l'economia, mentre la sfera domestica continua ad essere il luogo per antonomasia ad esse riservato.

\footnotetext{
${ }^{1}$ Data di ricezione: $10 / 11 / 2019$.

Data di accettazione: 10/11/2019.

${ }^{2}$ Docttore in Filologia Italiana, membro del Gruppo di Ricerca "Escritoras y Personajes Femeninos en la Literatura”, Universidad de Salamanca, Spagna; $₫$ angeloazzilonna@ @otmail.com.
} 
Partendo da simili considerazioni risulta ancor più attuale l'edizione critica "Escritoras de la Modernidad (1880-1920). La transformación del canon", che in un'ottica interdisciplinare e trasnazionale, raccoglie vite e opere di coraggiose scrittrici a cavallo tra il XIV e il XX secolo, uno dei momenti storici più delicati che segna l'inizio di una nuova modernità, innescando la prima ondata storica del femminismo. Proprio la cultura, l'istruzione e la scrittura tradizionalmente negate alla donna, considerate dalla vetusta società maschilista fuorvianti se non elementi sovversivi, in quanto prodromi di una "pericolosa" emancipazione femminile, costituiscono il fulcro dell'interessante miscellanea in cui si da spazio alla penna di intellettuali di ogni latitudine, di pregevole fattura estetica ed enorme utilità per un effettivo progresso civile, stigmatizzando la condizione storica di marginalità culturale e socioeconomica della donna.

L'"inusuale" connubio donna-scrittura funge da motivo conduttore delle 164 pagine che compongono i diciotto capitoli nei quali letterate di tutto il mondo testimoniano e denunciano limiti, coercizioni ambientali e pregiudizi di cui erano vittime. Tuttavia, al contempo, si soffermano sulle enorme potenzialità artistiche, sulla forza morale e sulla lucidità intellettuale con cui prendere coscienza e divulgare in tempi non sospetti circa la loro iniqua posizione nelle rispettive società di appartenenza, per poi intraprendere lotte individuali e collettive, volte a favorire quel fondamentale processo di sensibilizzazione dell'opinione pubblica, partendo sia dalle proprie vicende di vita privata, plasmate in accorate pagine biobliografiche, sia da avvincenti fictions narrative di donne coetanee, ispirate dalla ostile realtà per la donna tra fine Ottocento ed inizio Novecento, raccontate sempre da una prospettiva femminile. Creare, ricostruire e preservare la memoria collettiva storico-sociale è uno dei più ambiziosi propositi del volume, nel quale si indaga ad esempio sull'origine e sulle conseguenze delle oggettive 
difficoltà, sulle prevaricazioni subite dalle donne, sulla loro problematica quotidianità, risultati di una retriva mentalità machista.

Il volume presenta opere di letterate di professione e autodidatte di lingua italiana, tedesca, francese, spagnola e inglese, accomunate dalla volontà di "sfidare" le convenzioni, le leggi e i costumi dell'epoca per smuovere le coscienze femminili e della società. Tuttavia, la loro scrittura, impavida perché educativa, al pari delle loro vite, durante le quali hanno combattuto attivamente per rivendicazioni e diritti delle donne (voto, aborto, divorzio su tutti), ha conosciuto una fortuna intermitente; di fatti si tratta, nella maggiorparte dei casi, di artiste dimenticate, escluse da manuali, antologie e riviste, o relegate tuttalpiù ad una fama caduca e passeggera, permanendo ai margini del canone e quindi della storia letteraria. La pubblicazione di "Escritoras de la Modernidad (1880-1920). La trasformación del canon", è parte integrante del progetto di ricerca, promosso dall'Assessorato all'Istruzione della Regione spagnola della Castilla y León e dai Fondi Europei per lo sviluppo Regionale (SA019P17) intitolato "Escritoras inéditas en español en los albores del siglo XX (1880-1920). Renovación pedagógica del canon literario”, coordinato da Milagro Martín Clavijo, esperta docente salmantina, impegnata nello studio di letterate inedite, di notevole impegno sociale, col fine di colmare, almeno parzialmente l'ingeneroso vuoto editoriale e pubblicistico nei loro confronti. "Escritoras de la Modernidad (1880-1920). La transformación del canon", pertanto, si configura come un volume nel cui titolo è insito un doveroso riconoscimento a tali scrittrici, pioniere ascrivibili alla prima e decisiva corrente femminista, modernamente intese, anche per quanto concerne generi, tematiche e stili letterari, che ha saputo analizzare, canalizzare e valorizzare il crescente desiderio di istruzione e cultura da cui sviluppare consapevolezze e opportune proteste nei confronti del sistema patriarcale del periodo; in esso, si denota un lodevole associazionismo e 
protagonismo femminile, sintesi di una apprezzabile capacità critica, di maggiore autocoscienza e orgogliosa dignità, consentendo alla donna di iniziare a varcare la penalizzante sfera domestica e provinciale, a cui sembrava condannata, per avvicinarsi finalmente ad ambienti ed ambiti urbani, cosmopoliti, pressoché sconosciuti.

Nella struttura complessiva del volume la letteratura italiana occupa un posto decisamente rilevante con svariati capitoli. Due di essi sono dedicati alla scrittrice Grazia Deledda. Nello specifico Piero Mura, nel suo contributo "Grazia Deledda o della labile immortalità" traccia un profilo della letterata sarda, caratterizzato da una femminilità ribelle e indipendente, dalla forte personalità, riottosa verso ogni tipo di coercizione ambientale e stigmatizzando l'oblio nel quale è piombata la sua opera che sfugge ad ogni canone ed etichetta e benché insegnita nel 1926 del Premio Nobel della Letteratura é stata relegata dalla critica a mera rappresentante del folclore della sua terra.

Nadia La Mantia continua lo studio della principale scrittrice sarda nel suo saggio “Superare le barriere dell'analfabetismo: la scrittura come strumento per il riscatto culturale e sociale in sardegna. Il vcaso di Grazia Deledda", risaltando il fondamentale ruolo dell'istruzione per il riscatto femminile e dell'isola natía, una delle aree geografiche con preoccupanti tassi di analfabetismo e abbandono scolastico.

Altra letterata italiana, Amalia Guglielminetti è oggetto di ricerca in due pregevoli lavori; Irene Scampuddu in "Il percorso letterario di Amalia Guglielminetti a cavallo tra il XIX e il XX secolo", evidenzia il suo lodevole autodidattismo e il compito affidato alla scrittura, preferito alla rivendicazioni di massa come elemento di rottura verso la dominante cultura patriarcale. Mercedes Arriaga Flórez in "Las vagabundas: Sibilla Aleramo y Amalia Guglielminetti", considera l'automarginalità della 
Guglielminetti come forma di libertà ed autocoscienza, criticata, al pari della scrittrice Sibilla Aleramo per la vita e l'opera così spiccatamente individualiste ed erranti, fuori dai canoni sociali e letterari, dove il viaggio diventa metafora di nuova esistenza, dinamica, intensa, aperta, opposta alla staticità e al bigottismo che rappresentava la casa, spazio chiuso, asfittico, identificativo della donna italiana di inizio XX secolo.

Due capitoli dell'edizione rimarcano la funzione didattico-pedagogica della letteratura e di alcuni generi nei quali si sono distinte alcune scrittrici italiane. In particolare la professoressa Sara Velázquez in "La pedagogía del relato o el relato de la pedagogía en Ida Baccini: escritora, periodista, maestra", si sofferma sui racconti per l'infanzia di Ida Baccini, risaltandone la centralità nella costruzione e nella rappresentazione dell'identità nazionale, in quanto veicoli ed espressioni di aspetti storico-sociali particolari di un determinato territorio con cui trasmettere modelli, valori, costumi, decisivi per "creare" una concetto di patria e di Italia.

Nella stessa scia si inserisce il saggio di Salvatore Bartolotta e Maria Gracia Moreno Celeghin intitolato "Los géneros de Annetta Vertua" nel quale ricostruisce la traiettoria letteraria di Annetta Vertua, contraddistinta da importanti finalità educative, come si evince dai suoi romanzi, dai suoi racconti per bambini e dai suoi manuali di comportamento in casa e in società, rivolti a ragazze e giovani signore. Al contempo, tali generi letterari rivestono una considerevole valenza non solo pedagogica e culturale ma anche linguistica, proprio nel delicato proceso dell'Unificazione italiana.

Un altro genere letterario in cui si sono cimentate in maniera originale e pioneristica alcune dimenticate scrittrici transalpine, ben analizzato nel lavoro di Yolanda Romano Martín è il romanzo poliziesco; infatti in "Escritoras y personajes femeninos en los albores del giallo italiano: Carolina Invernizio, Matilde Serao y 
Erminia Bazzocchi”, se da un lato si conferma il topos che vuole la donna vittima indifesa di violenze fisiche, sessuali e psicologiche, dall'altro lato si scorge una nuova speranza, prodotto di una più equa mentalità per cui spesso è la donna stessa che si erge a "giudice", a investigatrice energica, risolutrice di delitti e misfatti. Invece, María Burguillos Capel nel suo contributo "La narrativa de Annie Vivanti: reescribiendo la imagen de la mujer-serpiente", offre una dettagliata e ragionata interpretazione di tre cruciali romanzi di Annie Vivanti, dove si descrivono abusi, orrori e depravazioni di cui però i personaggi femminili rivestono un ruolo di agente attivo, anche in negativo, mediante condotte indolenti, ciniche ed amorali, paragonabili a quelli maschili. Si presentano donne intraprendenti, audaci amanti, capaci di sedurre non solo con la belleza estética bensì col carisma, sconfessando e trasgredendo il canone letterario, riflesso dell'ordine etico e sociale vigente. Nella stessa direzione e con la medesima intenzione di destrutturare il topos sul femminile si muove il contributo di Milagro Martín Clavijo, "La narrativa de Anna Franchi a caballo entre el siglo XIX y XX: los relatos de Dulcia Tristia" concedendo visibilità ad una scrittrice pugnace, esclusa dagli studi letterari, eppure meritevole di attenzione proprio perché denuncia la marginale condizione della donna nel suo riduttivo ruolo di "angelo del focolare domestico"; nei suoi romanzi si esorta la donna a superare la dimessa e conseziente passività verso l'uomo, per costruire autonomamente il proprio destino, cercando una armoniosa e completa realizzazione personale e professionale, magistralmente condensata nel binomio letteratura-amore con cui esprimere pienamente qualità artistiche e capacità lavorative.

Un'ennesima scrittrice combattiva, caratterizzata da ammirevole forza di spirito e da ferme convinzioni morali è Ada Negri alla quale Antonella Cagnolati dedica il capitolo "Poetizando su propia vida: Fatalità e tempeste de Ada Negri”. Particolarmente 
apprezzabile risulta la condanna nei confronti dei vizi, della corruzione e del cinismo amorale della società italiana del periodo, personificata dalla classe borghese a cui la letterata oppone dicotomicamente l'umile, ingenua e oppressa classe operaia che trova ribalta e dignità poetica.

Uno degli ambiti letterari in cui le giovani scrittrici si ritagliano importanti spazi è sicuramente quello delle riviste e dei periodici che agli albori del Novecento registra una forte presenza e protagonismo al femminile, con donne nelle vesti di direttrici e/o collaboratrici regolari, segnale inequivocabile del graduale cambiamento socioculturale. In tal senso è perfettamente esemplificativo lo studio di Barbara Meazzi "Non sarà un giornale femminista?". La Donna-Rivista quindicinale illustrata (1905-1968)" in cui si sintetizzano le tematiche principali affrontate nella rivista, quali il suffragismo e più in generale l'emancipazionismo, sensibilizzando lettori e lettrici sulla annosa questione femminile, battendosi per l'accesso all'istruzione, per il diritto alla formazione, per l'uguaglianza politica, giuridica e civile delle donne italiane. Analogamente, in un altro capitolo del volume firmato da Claudia Adriana López Ramírez, dal titolo "Revistas decimonónicas en México, la presencia femenina y el canon literario" enfatizza il ruolo esercitato dalla rivista "La Siempre Viva" che ebbe il merito di promuovere la parità culturale e letteraria tra uomo e donna in terra messicana.

Il volume miscellaneo, infatti, evidenzia la diffusa e penalizzante negligenza, mista a indifferenza da parte della critica letteraria internazionale verso le donne che con qualità artistiche e coraggiosa determinazione, in tempi non ancora maturi, cercarono di dedicarsi e affermarsi nel campo della letteratura. In Italia, ad esempio, grazie ad un rivelatore studio realizzato da Maurizio Capone dall'eloquente e beneaugurante titolo "Verso un canone plurale. Le scrittrici nella critica di Giuseppe Antonio Borgese", contrassegnato da una sottile polemica, si analizzano metodi, 
parametri e pregiudizi sessisti con cui la critica ufficiale giudicava e sottovalutava le penne femminili, associando il loro eventuale successo a periodi di decadenza storica e letteraria, partendo dal postulato secondo il quale le donne capterebbero in ritardo idee e peculiarità stilistico-tematiche dei vari movimenti culturali. L'oblio e la scarsa valorizzazione verso le donne letterate, in Inghilterra riguardano la scrittrice Charlotte Mary Yonge alla quale Alejandro Camacho González dedica un accorato capitolo "Charlotte Mary Yonge y el canon: análisis de un protagonismo y de una ausencia", nel quale rende omaggio alla colta e prolifica artista anglosassone, gran conoscitrice della cultura spagnola ed europea, offrendo una documentata esegesi sull'esclusione letteraria della sua opera in prosa che aveva nelle giovani donne le loro principali destinatarie.

Tuttavia, benché in maniera sporadica e tardiva, la critica letteraria ha riscoperto e apprezzato la talentuosa produzione di altre scrittrici. In tale ottica, restando nell'ambito della lingua inglese, ma di area nordamericana, nel capitolo "Kate Chopin inédita: «La Tormenta» en el canon literario norteamericano", la professoressa Teresa Gisbert si concentra su un breve racconto dell'autrice ("La Tormenta") che a suo tempo suscitò vibranti strali polemici per la presenza di elementi erotici e adulterini della protagonista, peraltro non adeguatamente "punita" in base al ferreo moralismo fine ottocentesco. Altrettanto lodevole e intrepida risulta l'opera di Cornelia Sorabji's, come ben illustra Jorge Diego Sánchez in "Contesting seclusion from letters: Cornelia Sorabji’s early writings on purdah (1902-1903) giacché denuncia il purdah indiano, ossia la reclusione delle donne nel pubblico e nel privato, nonché le ingerenze politiche che la motivano.

L'anelito emancipazionista femminile e la successiva valorizzazione da parte della critica riguardano, infine, le opere di due scrittrici di Germania e Spagna che arricchiscono la conoscenza del contesto letterario continentale tra il XIX e il XX secolo. Si tratta dei capitoli degli esperti studiosi dell’Università di Salamanca Juan 
Manuel Martín Martín e María Jesús Framiñán de Miguel. Il primo, nello specifico, nel suo contributo intitolato "La imagen de la escritura y los conflictos femeninos en la obra de Emmi Lewald", indica la lenta ma inarrestabile consapevolezza e la progressiva autorevolezza della donna tedesca che desidera e conquista una certa indipendenza coniugale, il diritto al divorzio, scorgendo nella letteratura la massima espressione della propria libertà, della creatività, della sensibilità e in definitiva della modernità. Ulteriore conferma circa la crescente autocoscienza femminile, con conseguente volontà di partecipazione attiva alla vita politica e culturale proviene dalla Spagna, dalle acute riflessioni di María Jesús Framiñán de Miguel nel capitolo "De la periferia al centro del canon: Lucía Sánchez Saornil en la antesala del 27”, dove traccia un profilo dell'opera poetica della "ultraista" e combattiva poetessa Lucía Sánchez Saornil che plasma nella sua lirica avanguardista, decisamente antiprosaica e anarchica, forti quanto incoraggianti messaggi in difesa dei diritti delle donne, soprattutto di quelle lavoratrici come le contadine spagnole, troppo soventemente in balia dello strapotere e del sopruso maschile, demitizzando al contempo il matrimonio, visto come un'istituzione che limita e inibisce le potenzialità e la loro piena realizzazione personale.

In sintesi, il volume Escritoras de la Modernidad (1880-1920). La transformación del canon presenta svariati e interessanti spunti letterari e critici che lungi dall'appartenere esclusivamente al periodo storico compreso tra Ottocento e Novecento sono atemporali, in quanto utili e validi anche per le donne e per la società attuale che hanno il diritto-dovere di conoscere e riconoscere le avversità, le battaglie, le iniquità e per questo i notevoli e conseguenti meriti umani, civili e artistici di scrittrici coraggiose e talentuose a cui né la storia né la critica letteraria ufficiale hanno saputo o più probabilmente voluto rilevare. In tale prospettiva, tale volume rappresenta non solo un tributo all'intrinseco valore poetico delle letterate, ma costituisce al contempo un 
piccolo e necessario tassello per una più ampia e paritaria ricostruzione storico-sociale e culturale del periodo in questione, da cui peraltro é più agevole comprendere le complesse e odierne condizioni femminili, riflesso a loro volta di anacronistiche e intollerabili anomalie discriminatorie socio-culturali. 